Research Article

\title{
Surface Waves Analysis of Efficient Underwater Radio-Based Wireless Link
}

\author{
Muhammad Rauf $\mathbb{D}^{1},{ }^{1}$ Atif Jamil $\mathbb{D},{ }^{2}$ Muhammad Dawood Idrees $\mathbb{D}^{3},{ }^{3}$ Arsalan Ansari $\mathbb{D},{ }^{1}$ \\ and Abdul Sami ${ }^{1}$ \\ ${ }^{1}$ Department of Electronic Engineering, Dawood University of Engineering and Technology, Karachi, Pakistan \\ ${ }^{2}$ Department of Computer Systems Engineering, Dawood University of Engineering and Technology, Karachi, Pakistan \\ ${ }^{3}$ Department of Industrial Engineering and Management, Dawood University of Engineering and Technology, Karachi, Pakistan
}

Correspondence should be addressed to Muhammad Rauf; muhammad.rauf@duet.edu.pk

Received 3 September 2021; Accepted 1 October 2021; Published 2 November 2021

Academic Editor: Dao B. Wang

Copyright (c) 2021 Muhammad Rauf et al. This is an open access article distributed under the Creative Commons Attribution License, which permits unrestricted use, distribution, and reproduction in any medium, provided the original work is properly cited.

\begin{abstract}
The domain of underwater wireless communication (UWC) link is gaining much attention due to an increase in various underwater activities such as offshore hydrocarbon exploration, underwater unmanned vehicles (UUV), and military practices. Increased bandwidth and a reliable data link are mainly required for such activities. Both requirements of the domain are heavily affected by the highly conductive property of the seawater. This paper demonstrates the performance evaluation of radiofrequency-UWC, focusing on surface wave analysis, to propose a reliable solution for offshore activities. A constructive interference scheme can be useful due to the sharp difference in the properties of the two mediums (air and seawater). To that end, an experimental setup is created, and a corresponding finite element method (FEM) based simulation of the radio-based wireless link is run. This is because it has higher bandwidth and speed than acoustic and optical approaches. A conduction current mechanism transmits and receives data in a synthetic water tank containing a prepared conductive media (saltwater). The study of changing depths of transmitter-receiver nodes in saltwater shows that surface waves cause significant noise reception in shallow water (less than dipole length, below water level). During a series of experiments in the tank, the lowest bit error rate (BER) is observed only when the node's submerged height was greater than dipole length. As a result, it is meant to provide a genuine data channel model. The discovery and analysis will aid in the development of a dependable underwater data link, with applications including shortrange diver-to-diver communication, and UUV capability.
\end{abstract}

\section{Introduction}

Due to the growing demand for underwater marine applications, developing an underwater wireless communication connection has become a fast-growing yet challenging research subject in recent years. As evidence, many papers $[1,2]$ offered underwater activities, including oil rig monitoring, offshore surveillance, port security, and geological studies, and associated expeditions solutions. However, due to substantial attenuation in the highly conductive medium, which restricts skin depth, existing models for wireless communication links in the air cannot be utilized underwater [3]. As a result, the underwater channel's bit error rate is significant, especially when the lateral communication extent is longer. In this scenario, a reliable communication link has become vital for several successful offshore operations, including controlling and monitoring UUV.

Acoustic, optical, and radiofrequency (RF) are the most frequent methods for building an underwater wireless network. However, all of the techniques have some advantages and disadvantages. For a long time, acoustic waves have been utilized as an authentic scheme. However, lately, they have been demonstrated to fail when used in shallow water. It is caused by reflections from the bottom and sea surface (reverberation effect). Furthermore, acoustic technologies have a sluggish data transfer speed. Backscatter 
from suspended materials and refracted light from the surface, on the other hand, can cause unreliability in optical techniques. In all circumstances, the surrounding medium parameters and wave penetration depth significantly impact an underwater channel link.

Furthermore, RF signal transmission is hampered by the saltwater environment, which also inhibits long-distance wave propagation. For the UUV application, a tether cable has been reported as an option for real-time surveillance. However, the complex offshore environment and nonlinear dynamics of the UUV have made the purpose more challenging. In comparison, the RF-based method ensures a high data rate (up to $100 \mathrm{Mbps}$ ) in a shorter range while less affected by the water turbidity and ambient acoustic noise. These attributes support the method for the real-time surveillance of UUV. To develop a radio-wavebased system, it is required to analyze the critical components for the performance index measurements that affect the channel. Along with coil, loop, and dipole antennas [4], the current conduction method has been reported for the underwater radio connection as a deployment-friendly means to generate radio waves. In several reports [4-6], the present conduction method was used to illustrate the propagation of electric fields in a conducting marine/water environment.

This section aims to briefly analyze the state of the art in underwater wireless links using surface waves and their challenges. In most related work, the challenges are the range of the link, reliable data transfer rate, and bandwidth capacity. To address these issues, two alternate approaches are usually utilized: (1) receiving RF signals at air while propagating at the seawater-air interface and then refract to the air path, followed by air to water signal propagation, due to a submerged transmitting source, and (2) utilizing the phenomenon of the guided wave at the air-seawater interface to build the wireless link. Several documents following both phenomena have been reported. A report where several orientations of submerged dipole source like horizontal magnetic dipole along $x$-direction (HMDx), horizontal electric dipole along $x$-direction (HEDx), vertical magnetic dipole (VMD), and vertical electric dipole (VED) has been compared. Among them, HEDx has exhibited the highest strength of the EM field that promises a more extended range of communication links with higher bandwidth $[5,6]$. Besides the horizontal range, the vertical range is also examined in some articles using surface wave analysis. A document claimed a vertical link range of $60 \mathrm{~m}$ using an applied source frequency of $10 \mathrm{kHz}$ [7]. Antenna impedance using a RF source demands impedance matching for a reliable link. Another work proposed an enclosed source (a monopole helix) in ionized water that claimed improved coupling of EM wave in seawater using a seawater-air interface. The work used $50 \mathrm{MHz}$ bandwidth and achieved an acceptable EM signal strength at a vertical offset of $5.5 \mathrm{~m}$ [8]. Utilizing the phenomenon of surface waves for achieving an effective link, an encapsulated underwater communication system is proposed. With this scheme, medium-to-high frequency is reported [9]. The common issue found in all related work is the difference in experimental and analytical outcomes in terms of range. In addition, BER and SNR are considered vital indicators for an efficient communication link. Due to the notable variation in the design schemes, both indicators are usually neglected.

Another crucial aspect of the underwater wireless communication link between two nodes is the modulation of signals based on transmitted frequency. A document used turbo code to address the scenarios to increase the signal-to-noise ratio (SNR) of the higher-order Phase Shift Keying such as 8PSK and Quadrature Amplitude Modulation systems such as 16QAM. The Orthogonal Frequency Division Multiplexing (OFDM) approach is capable of amplitude variation (reported as $20-25 \mathrm{~dB}$ ) when applied to a frequency-dependent channel [10]. PSK and Complementary Code Keying (CCK) were compared to data rates utilizing IEEE $802.11 \mathrm{~b} / \mathrm{g}$ in such a way that BPSK and QPSK displayed increased error protection at the expense of reduced transmission speed when analyzing various modulation methods. The ON-OFF shift keying modulation method also claimed a $1 \mathrm{Mbps}$ transmission rate while increasing the lateral propagation range [11]. Digital signal processing was incorporated using a comparable method, resulting in a transmission range of $50 \mathrm{~cm}$ at a data rate of 1 Mbps. Power Spectrum Density (PSD) measurement of the underwater link utilizing Amplitude Shift Keying (ASK) at frequencies ranging from 3 to $10 \mathrm{kHz}$ and PSK at $8.66 \mathrm{kHz}$ [12] validated real-time data transmission. Software-defined radios, digital modulation at $2 \mathrm{Mbps}$, and a signal frequency of $2 \mathrm{MHz}$ can easily achieve a radio link range of $2.2 \mathrm{~m} \mathrm{[13].} \mathrm{Furthermore,} \mathrm{for} \mathrm{underwater} \mathrm{radio}$ communications, multiband carrier modulation has revealed some transceiver setup constraints [14]. In addition, the flexible modulation technique employing Phase Locked Loop (PLL) is employed to estimate the BER at various frequencies to study the aforementioned restrictions [15]. Additional studies have been written on how to improve the underwater electromagnetic wave communication model by using directed antennas, angular movement variations, and other modulation techniques and reducing path losses $[16,17]$. Following a review of the several modulation techniques used in underwater communication systems, it was discovered that modulated signals are frequently evaluated as functions of frequency, source-receiver offset, and associated noise. However, it should be noted that a long radio link range necessitates a wide antenna and a high power output. 2ASK modulation (or OOK modulation for digital modulation) has proven its efficiency in circuit simplicity and design implementability within the given limits [16]. Aside from modulation techniques, there is a wealth of information available on underwater antenna design. The performance and usefulness of existing designs such as dipole, loop, and coil antennas are presented [18]. Our most recent work explored frequency variation using 2 ASK modulation in relation to the probability density function, as shown in Figure 1. The technique is used to see if the channel is stable and if larger data samples are feasible. The encouraging finding revealed that a transmitter and receiver length of $20 \mathrm{~cm}$ could efficiently receive a $100 \mathrm{kHz}$ signal [19]. 


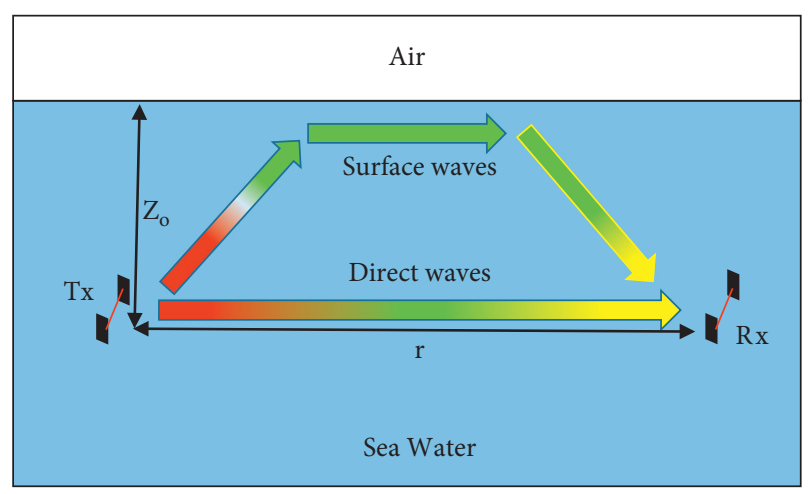

FIGURE 1: Channel architecture.

\section{Problem Statement and Proposed Solution}

For seawater as the surrounding medium, the wireless link has to rely heavily on its physical and electrical properties. The conductive medium usually hinders the waves from traveling longer but also causes such interference that affects the link reliability. Therefore, it is known that the successful communication link depends solely on a maximum communication range. This implies that if the range of a UUV (primary node) exceeds the range of the secondary node, then the communication will not be established. This binary approach usually fails in practical offshore practice due to signal reflection from either seabed or sea surface. In this work, we consider the problem of the unreliable wireless link between two nodes for the UUV application. It has been demonstrated in the literature that the surface wave may assist in obtaining a higher magnitude of the received signal due to the guiding factor between two mediums. Using this situation, we proposed a solution of surface waves evaluation for establishing a reliable communication link. BER is practiced as a critical indicator while examining the surface wave for a successful link.

In this work, the system design uses a current conduction mechanism to produce data signals in seawater (which mimics a highly conductive medium) in a synthetic water tank. A pair of identical copper electrodes are used to generate and receive the signals in the experiments. The design is characterized by Amplitude Shift Keying (2ASK). The electrodes are then used to send the modulated data signal. Receiving electrodes capture the electric field at the receiver end. To match, a demodulator circuit is designed and implemented. Most past research on the experimental design technique has focused on node-to-node wireless communication networks beneath the sea. However, the effects of surface waves on the receiving node are usually neglected. This paper examines the characteristics of surface and direct waves to create an efficient channel model.

\section{EM Modeling for Conductive Medium}

A conductive medium like saltwater shows ohmic losses due to its high permittivity and conductivity, limiting the spread of electromagnetic waves in the medium [20,21]. Numerous solutions have been presented, including a model for wavelength-compensated path loss with a range of $50 \mathrm{~m}$ and an attenuation of $140 \mathrm{~dB}$ [22]. When waves propagate in seawater, a high absorption loss is a stated fundamental property of the medium. On the other hand, waves traveling through the air-seawater interface are likely to be refracted. It is well established that a considerable number of the transmitted waves are guided down to the receiving antenna via a low loss channel (the boundary). The phenomenon is depicted in Figure 1. Similarly, a wave propagation modeling at the seawater-seabed interface can be monitored for the deeply submerged transceiver [23]. The sudden change in conductivity at the seawater-air interface instead of the seawater-seabed interface distinguishes the two events. As a result, this paper examines a possible channel model for the shallow submerged transceiver by varying the propagation depth.

Mathematical modeling enables the computer simulation to characterize and analyze experimental data, culminating in creating an optimal design performance index. Experiments were conducted in a synthetic water tank utilizing a dipole source and current conduction to generate EM waves. EM waves propagate at a low frequency in an underwater medium to simulate a higher skin depth [24]. As a result, only conduction current will be employed to approximate Maxwell's equations. The electromagnetic field generated by a sufficiently narrow finite conduction current generated by horizontal electric dipoles in a homogeneous, time-invariant conductive medium is given by

$$
E_{r}=\frac{I_{o} d \cos \theta}{2 \pi(\sigma+j \omega \varepsilon) r^{3}}(1+\gamma r) e^{-\gamma r},
$$

where $d$ represents the literal gap between transmitting electrodes, $I_{o}$ represents the applied current, $r$ represents the offset between transmitting and receiving electrodes, $\omega$ represents the angular frequency, $\varepsilon$ represents electrical conductivity, and $\mu$ represents magnetic permeability [25]. Due to the significant attenuation of waves passing through conductive saltwater, the propagation coefficient is defined as

$$
\gamma=\sqrt{-\varepsilon \mu \omega^{2}+j \omega \mu \sigma}
$$

While shallow water concentrates wave propagation, waves traveling over saltwater may reach the sea surface [26]. Using the propagation constant, a model of airwaves (surface waves) travel at the interface of air and seawater picked up by the receiver is given by [27]

$$
E_{r}^{\text {Airwave }}=\frac{I_{0} d \cos \theta e^{\left[i k\left(2 Z_{0}\right)\right]} e^{\left(i k_{0} r\right)}}{2 \pi \sigma_{1} r^{3}},
$$

where $k=\left(i \omega \mu_{0} \sigma_{1}\right)^{1 / 2}$ is the complex low-frequency wavenumber associated with seawater and its conductivity and $k_{0}=\omega\left(\mu_{0} \varepsilon_{0}\right)^{1 / 2}$ is the wavenumber associated with air.

The final model for electric field intensity used in this work can be obtained as 


$$
E=\frac{I_{0} d}{2 \beta\left(2 r+3 Z_{0}\right)} e^{-\alpha\left(2 Z_{0}+\sin \theta Z_{0}\right)} e^{-j \beta\left(2 Z_{0}+\sin \theta Z_{0}\right)}
$$

\section{Methodology}

The underwater data connection link will be investigated at various depths in this study. The development of specialized hardware is used to accomplish this. The experimental setup consists of three essential components: copper electrode, transmitter node, a conductive water tank, and a copper electrode receiver node. The transmitter node oversees signal production, modulation, and eventually electrode connection. The node is made up of a Microcontroller Unit (MCU) that generates low-frequency data and a high-frequency carrier wave in accordance with the Serial Universal Asynchronous Receiver Transmitter (UART) protocol utilizing the 2ASK modulation technique. In an electrodebased similar scheme, theoretical displacement prediction is positively correlated with the experimental circuit design [28]. In our work, the modulator circuit is composed of active (triodes) and passive (resistors and capacitors) components used to modulate and enhance the signal. Assume that $V_{\text {in }}$ is the voltage at the circuit's input, $V_{t}$ is the voltage at the triode, and $K$ is the triode's constant ( $K=$ the ratio of the triode's and transformer's magnification). The calculated current flowing via the transmitting electrode $I$ is as follows:

$$
I=K \frac{\left(V_{\text {in }}-V_{t}\right)}{R} .
$$

A carrier frequency of $100 \mathrm{kHz}$ was used in these experiments. The frequency variation generated by the HED source was recently assessed regarding the probability density function, as shown in Figure 2. In addition, the method is used to determine the channel's stability and the feasibility of larger data sample sizes. With a transmitter and receiver distance of $20 \mathrm{~cm}$, the encouraging result revealed that the $100 \mathrm{kHz}$ signal could be received efficiently.

Subsequently, the conditioned signals are coupled with the submerged transmitting electrodes in a prepared $4 \mathrm{~S} / \mathrm{m}$ saltwater. The instrumentation, including the modulator and amplifier, enhanced the signal once the receiver electrodes received it. After removing noise from the data using a low pass filter, the amplified signals are demodulated to form the received 2ASK signal. Finally, the processed signal is compared to the reference voltages, and a comparator circuit outputs the result. Initially, the distance between the transmitter and receiver electrodes is set to $20 \mathrm{~cm}$, and the spacing between the transmitter and reception electrodes is maintained to $80 \mathrm{~cm}$. The components of the overall architecture for inspecting the data transmission link in increasing degrees of depth are shown in Figure 3.

The experimental tank has been set up to accommodate a wide range of components required for in-depth research and investigation. To develop a variety of experimental setups, the transmitter-receive offset, the space between transmitter and receiver electrodes, and the depth of both sets can all be varied. The experimental tank is depicted in

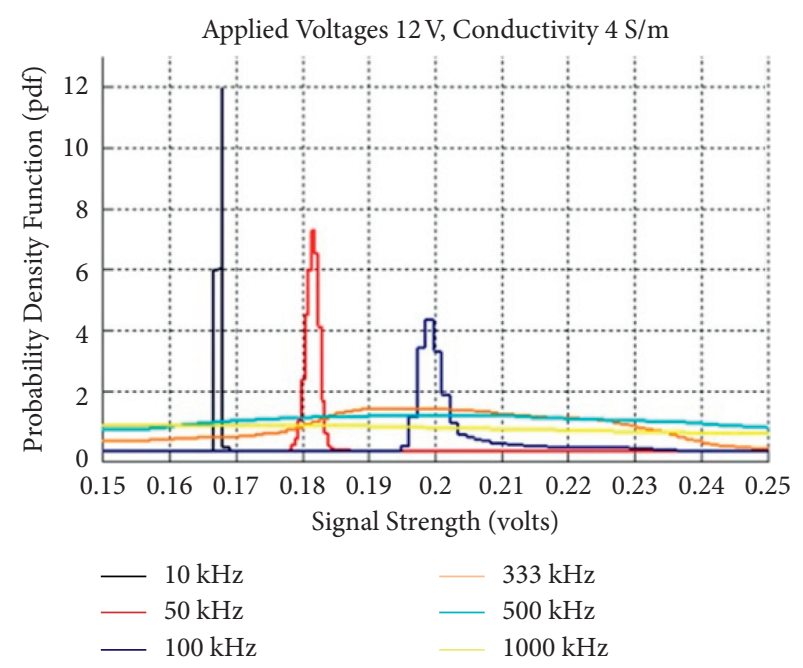

FIgURE 2: Plot of the received signal strength against the probability density function at frequencies from $10 \mathrm{kHz}$ to $100 \mathrm{kHz}$ [19].

Figure 4(b), while the corresponding simulation model is depicted in Figure 4(a). The simulation model, which is based on FEM, mimics the experimental design by placing six additional receivers on the seawater's surface to investigate surface waves while changing the transmitter's depth.

\section{Simulation Results}

The experimental tank is set up to examine and study a wide range of factors in detail. Variable transmitter-receiver offset, a separation between transmitter and receiver electrodes, and both sets' depth help generate a wide range of experimental combinations. The FEM-based simulation model replicates the experimental concept to investigate surface waves. The model is simulated using a commercially available 3D EM solver (CST Studio Suite ${ }^{\circledR}$, 2012). A highperformance computing machine contains 8 cores, 16 threads, $32 \mathrm{~GB}$ RAM. Each run of CST simulation took around 07 minutes.

From the FEM-based simulation of the corresponding experimental design, it is observed that the field response dropped down while varying $Z_{0}$ (the transmitter-receiver offset remains constant), demonstrating the impact of surface waves. Surface waves affected the signal attenuation at the receiver abnormally. At the end of the receiving electrodes, the attenuation was drastically high whereas, at the center of the electrodes, the decay is considerably low, as depicted in Figure 5. Based on the observation, this study will now investigate the amplitude of the signals at the surface of the saltwater. The potential distribution of the electric field at the surface is presented in Figure 6. The left column of Figure 6 shows the numerical solution's response utilizing 06 receivers (surface waves), while the right column shows the side view of the computed model, which reveals the depth variation of the dipole source. As the transmitter source was lowered from 10 to $40 \mathrm{~cm}$ below the sea surface, the potential dispersion of the electric field decreased. 


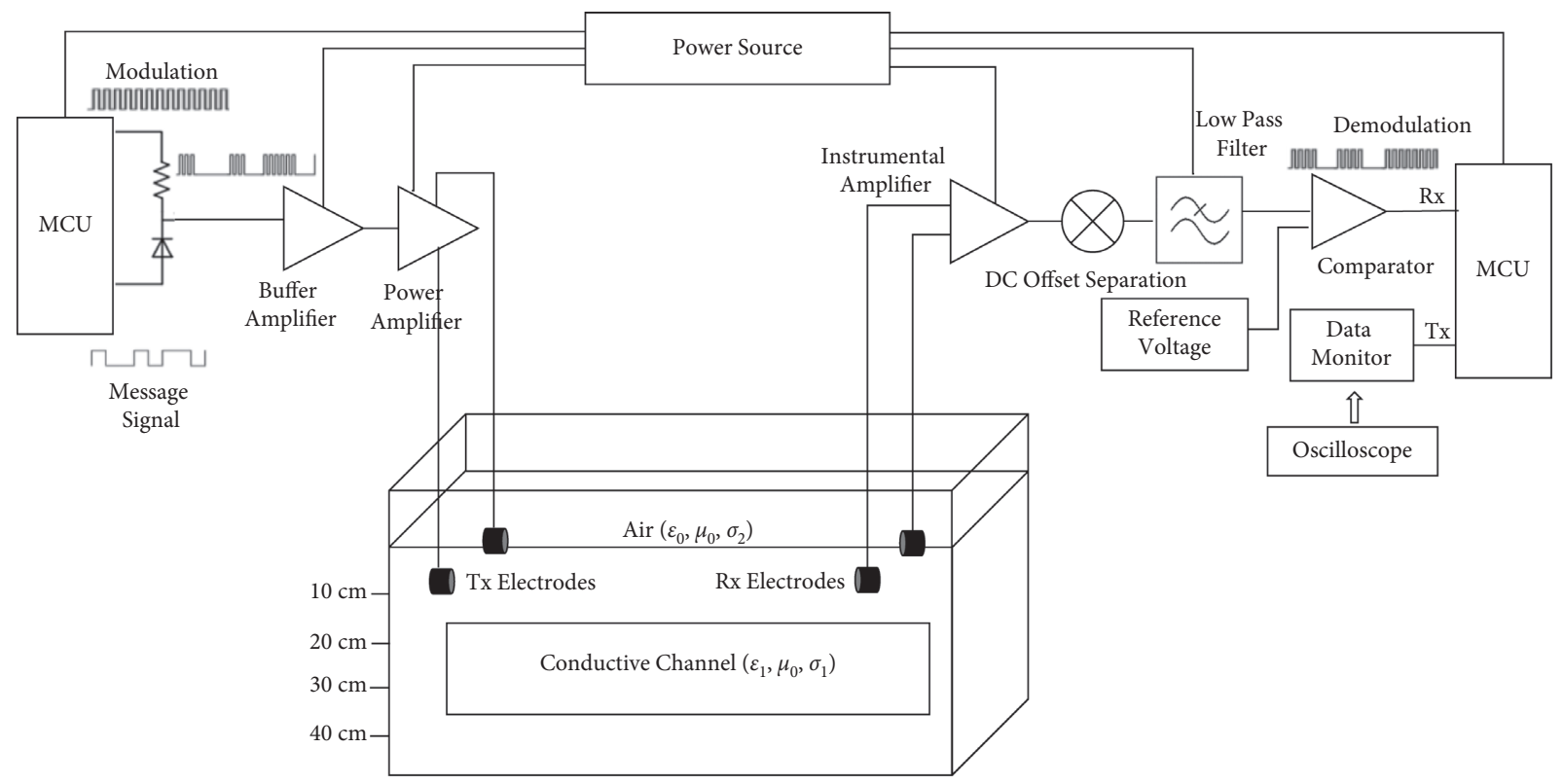

FIGURE 3: Block diagram for proposed system (inspired from [29]).

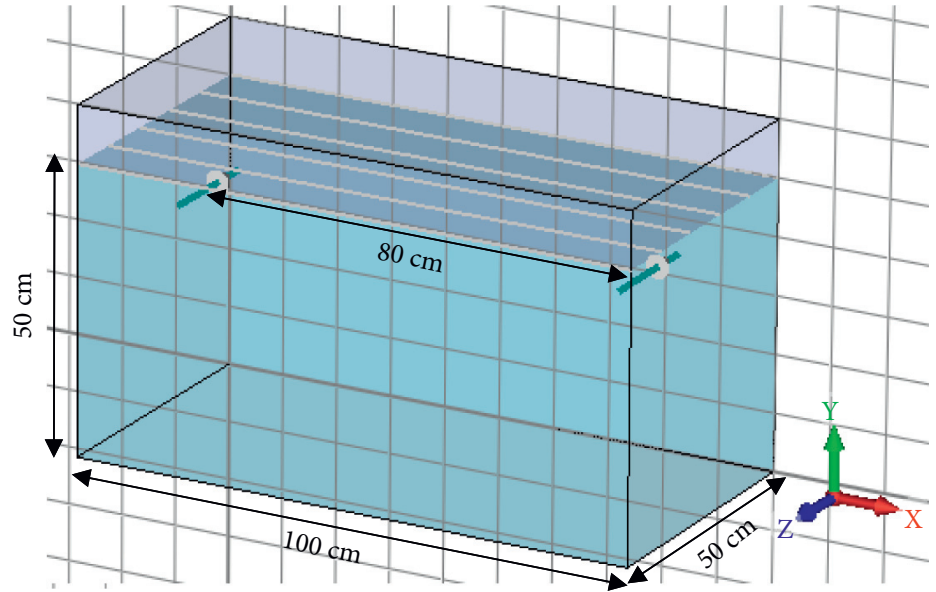

(a)

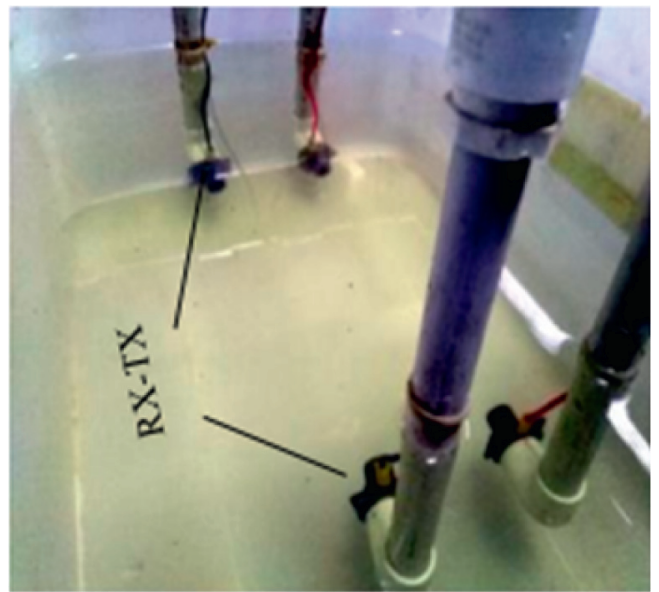

(b)

Figure 4: (a) Virtual model with receivers, immersed dipole source. (b) Top view of the experimental tank with transmitter and receiver electrodes.

The received signal intensity is shown in Figure 7 due to the depth variation in the $y$-direction, along the centerline between the transmitting and receiving electrodes. Even when the transmitter-receiver offset and injected power are constant, the responses displayed in Figure 6 for the surface wave and Figure 7 for the receiver electrode show a similar, falling signal strength trend. As a result, the receiver's falling trend in Figure 7 proves the superimposition of surface and direct waves. The data implies that the signal strength is higher at the shallower location of the transceiver system. Techniques such as BER and SNR, on the other hand, are mainly responsible for evaluating the communication channel's efficiency. In this study, we conducted an experimental analysis to determine the BER at various levels.

\section{Experimental Results}

Due to the dependence of received signal intensity on water depth, this article discusses establishing an experimental communication link under the same conditions. First, the active and passive circuitry of the front-end system is built in the way shown in Figure 3 to offer dependable and effective underwater communication. Then, in connection to the bit error rate of data transmission, extensive tests with changing the source-receiver depth were carried out and analyzed. Figure 8 shows experimental data reception using a small finite spread of known data packets over a $0.6 \mathrm{msec}$ time after 2ASK demodulation. An amplifier and a comparator's outputs are also included in the received signal. Thus, only 


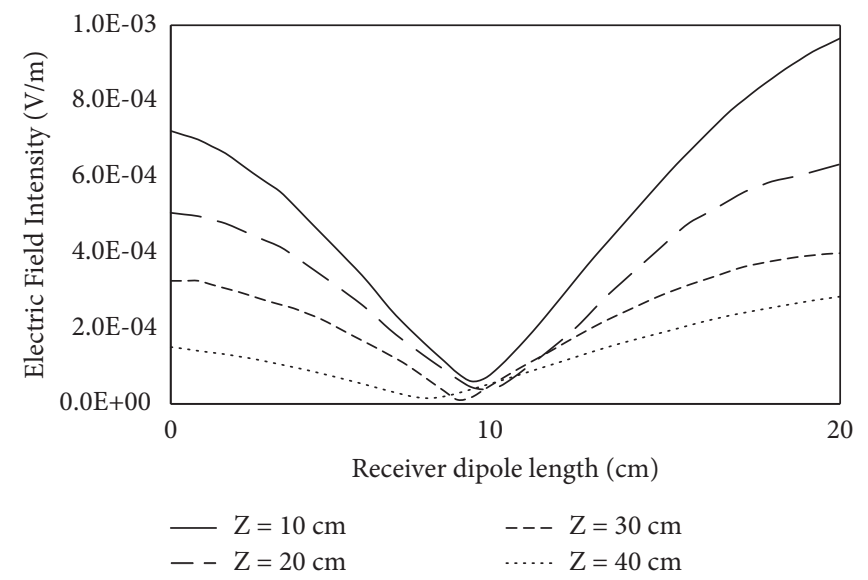

FIGURE 5: Electric field distribution along dipole length of the receiving electrode with different water depths.
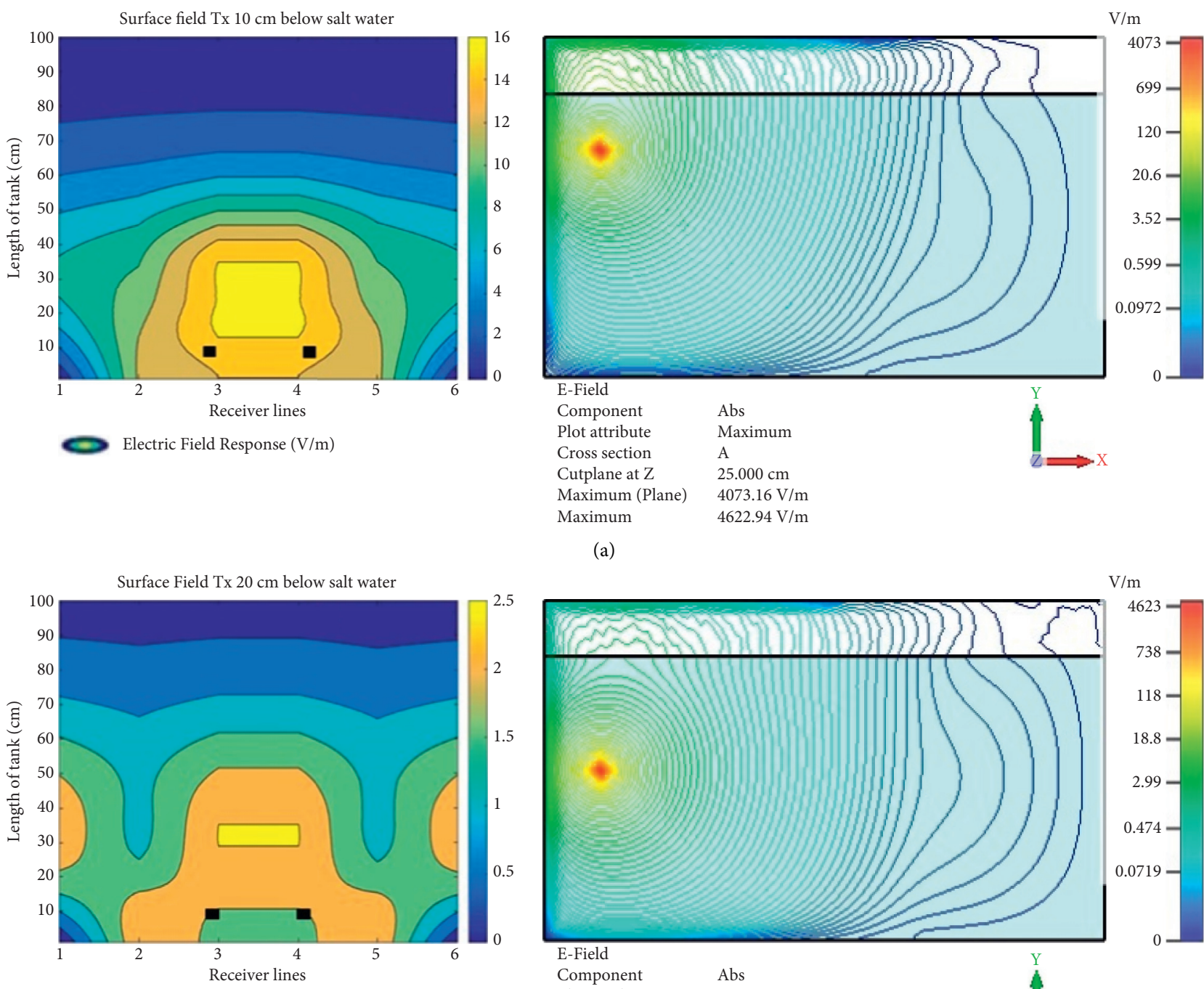

(a)

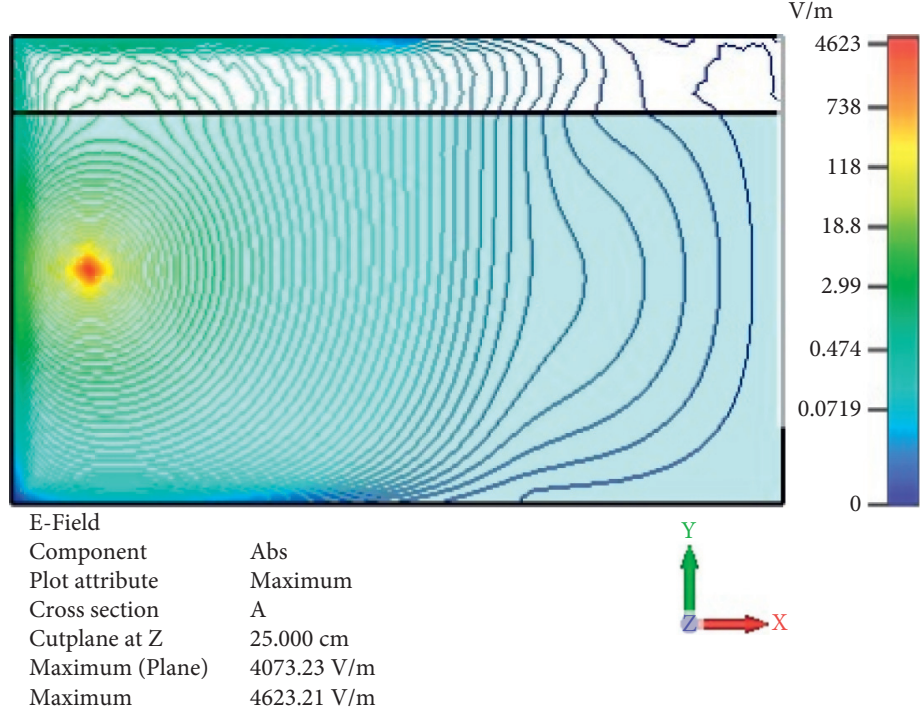

(b)

Figure 6: Continued. 

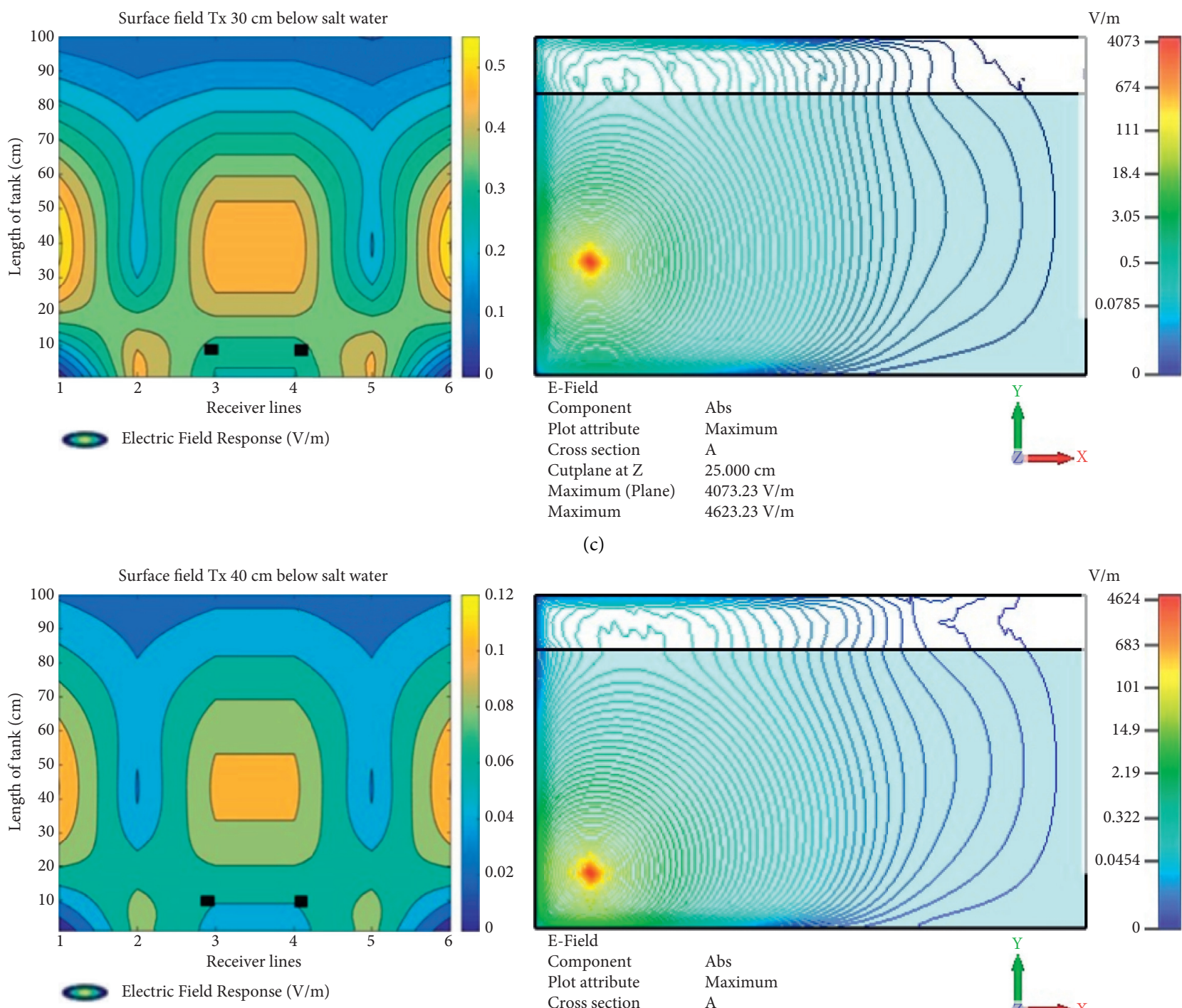

(c)

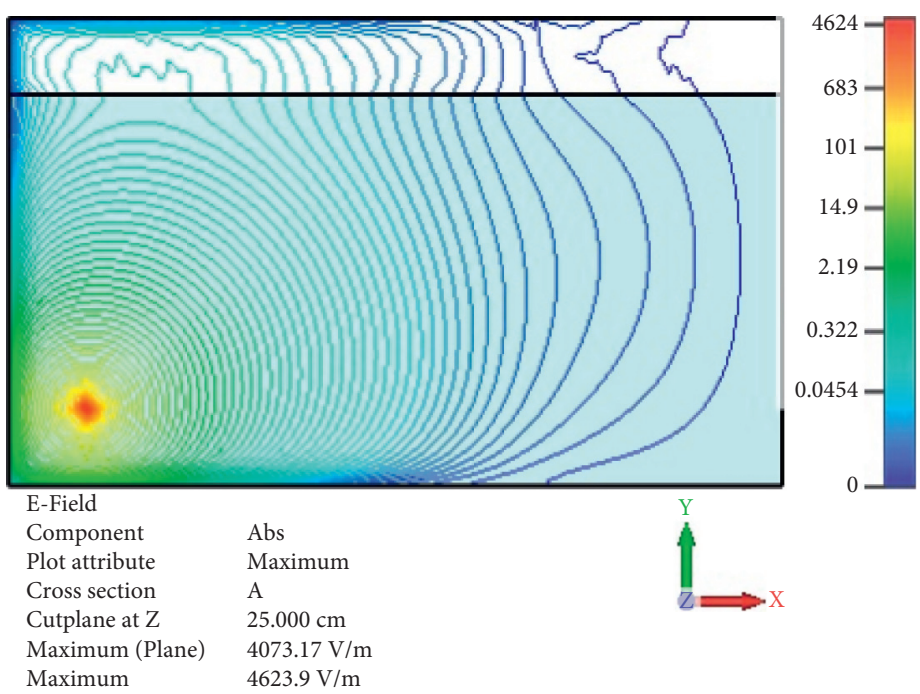

(d)

Figure 6: Transmitter positioned (a) $40 \mathrm{~cm}$, (b) $30 \mathrm{~cm}$, (c) $20 \mathrm{~cm}$, and (d) $10 \mathrm{~cm}$ below the seawater surface.

the source-receiver, $30 \mathrm{~cm}$ below the ocean's surface, is visible in the data.

Finally, when the depth of the transmitter source is altered, the BER is measured and analyzed for evaluation and conclusion. The electrode separation between the transmitter and receiver is $20 \mathrm{~cm}$, with an offset of $80 \mathrm{~cm}$ between the transmitter and receiver. With the chosen frame size, bit errors are calculated for each relevant data packet transfer depth. Figure 9 shows a simplified experimental way of estimating the BER. A frame header is used on the transmitter side to encode and transmit known data in hex format. The MCU's UART is used to access the decoded frame header on the receiving end. In Figure 10, the ratio of transmitted to received bytes in a data frame is calculated and shown.
The transmission network's effectiveness is determined using the BER shown in Figure 10. The measured BER is much higher when the transmitter source is $10 \mathrm{~cm}$ below the water surface than $30 \mathrm{~cm}$ below the water surface. $10 \mathrm{~cm}$ below the water surface is clearly a shallow water link where the magnitude of the received signal was observed highest, as presented in Figure 7 . The total water depth was $50 \mathrm{~cm}$ that outcast the possible effect of the bottom reflection of signals. This is also evident in Figure 7 where the signal strength is weakest at $40 \mathrm{~cm}$ below water level. In this situation, this study aims to determine if surface waves may be used to amplify the electromagnetic field. However, when the efficiency of a communication link is tested, it exhibits abnormal behavior. This realistic uncertainty has paved the way to conclude the effect of destructive interference by the 


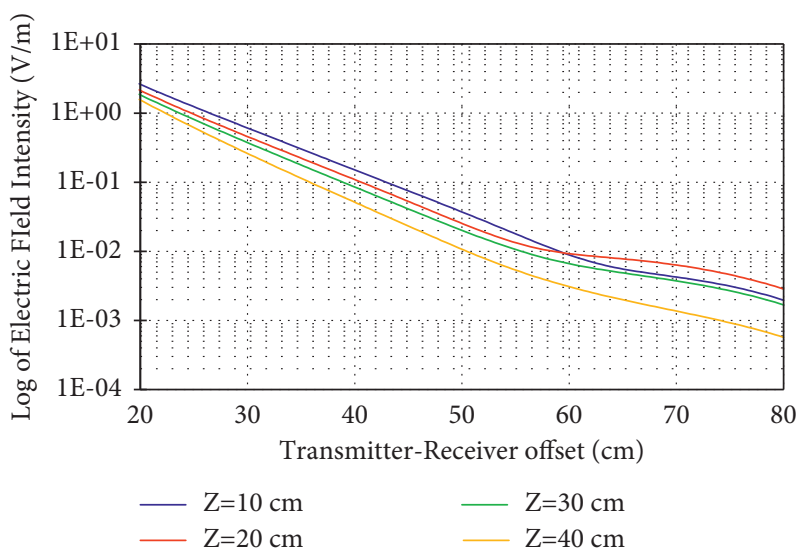

FIgURE 7: 1D electric field strength versus offset response by submerged transceivers with variable depths.

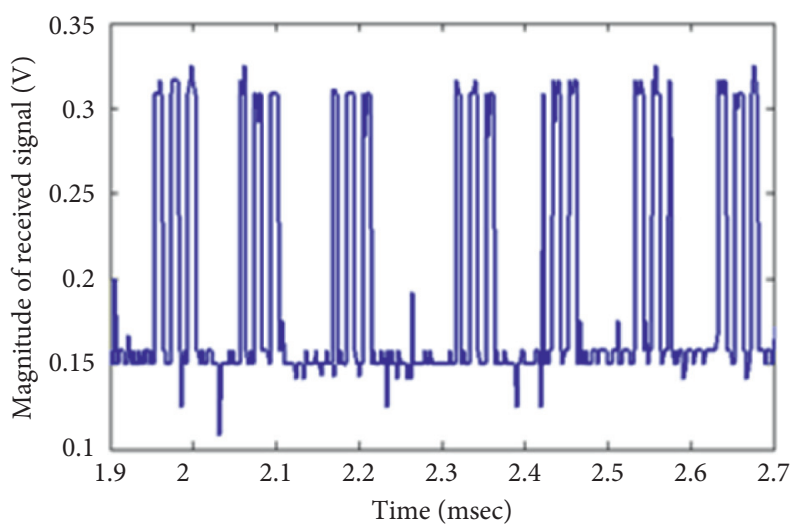

Figure 8: Received signal modulated with 2ASK.

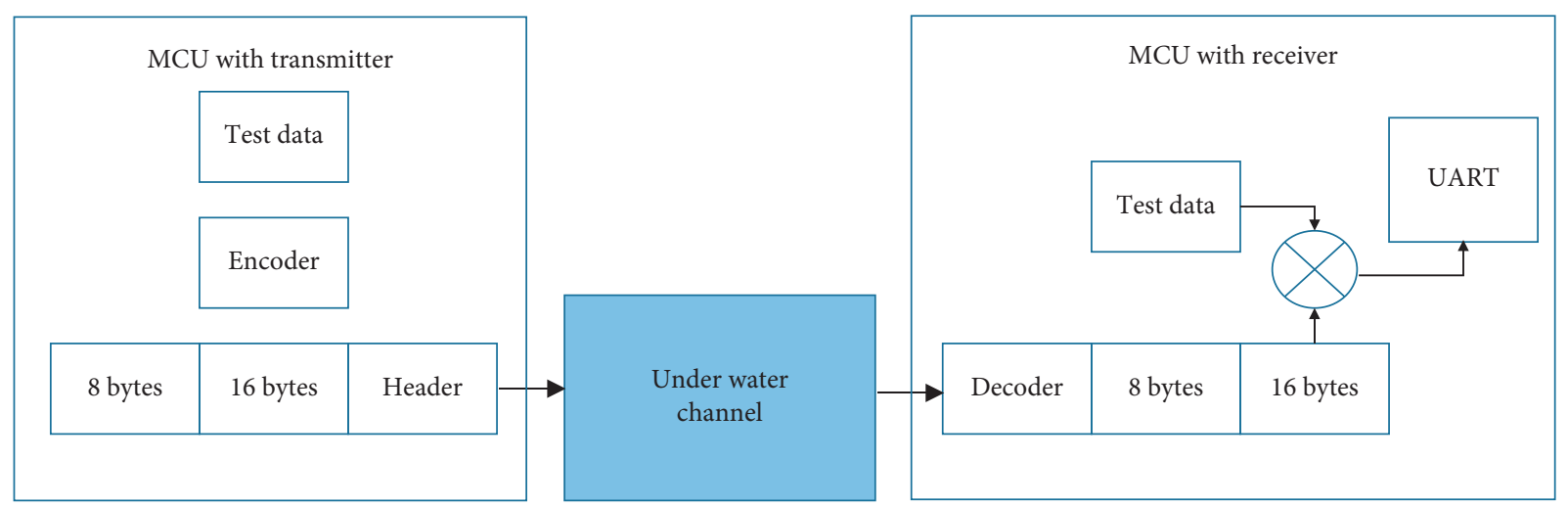

FIGURE 9: A scheme for examining the BER experimentally.

surface waves. As a result of this work, the lowest BER has been determined while the transmitter is positioned $30 \mathrm{~cm}$ below the water's surface. This depth is greater than the $20 \mathrm{~cm}$ dipole length.

Surface waves have a significant effect due to reverberations at the air-saltwater interface, as modeling and studies have shown. Apart from the influence, the horizontal extent of the wave is well known to be a function of skin depth. In a complex but straightforward relationship, the size of the transmitted wave is related to the frequency variation. As a result, it is assumed that frequency fluctuations contribute equally to direct and surface waves if the dipole source length remains constant. As a result of our research, we discovered the impact of surface waves on underwater data reception and a potential application for short-range diver-to-diver and UUV applications. 


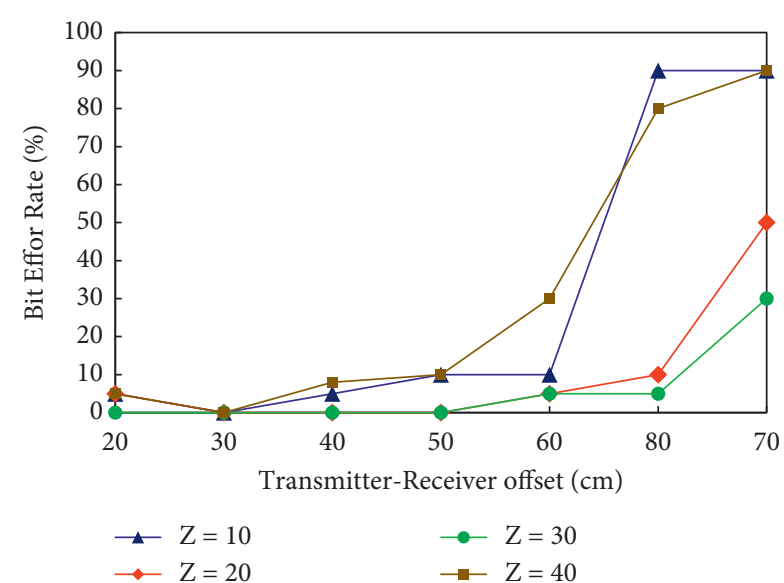

FIGURE 10: BER while varying depth of transmitter node at fixed baud.

\section{Conclusion}

The conduction current technique is adopted in this paper to create the underwater communication link focusing on UUV applications. This research aims to examine wave propagation behavior in the varying water column and its influence on the establishment of successful wireless links. An FEM-based simulation is conducted for the surface wave analysis where the depth of the transmitter is focused for this evaluation. Shallow water amplifies the received signal in both the near and far fields of the generated carrier signal containing data. However, submerging the transmitter to a depth of $30 \mathrm{~cm}$ produced reliable results in terms of BER, a fundamental parameter for evaluating communication links. Using the conduction current technique, this depth is calculated to be more than the dipole's length, which is around $20 \mathrm{~cm}$. As a result, this study concluded that the most viable technique to establish an underwater communication link is to reduce surface waves, possibly at a depth more significant than the dipole length. This scheme will lead to developing a reliable communication link between the unmanned underwater nodes. Furthermore, efforts such as injected power augmentation may be considered to extend the investigation to a more extended link. Additionally, instrumentation and structural modifications to the receiver may be investigated to enhance the receiver's ability to detect weak electric signals and reduce noise.

\section{Data Availability}

No data were used to support this study. The code used for the MCU to run is not available in the manuscript. The corresponding author may provide it upon request to assist the scientific community and readers.

\section{Conflicts of Interest}

The authors declare that they have no conflicts of interest.

\section{Authors' Contributions}

All authors contributed equally to this work.

\section{Acknowledgments}

The Higher Education Commission (HEC) Pakistan financed the research's experimental efforts (Grant no. 1710/ SRGP/R\&D/HEC). Besides, the authors are thankful to Dr. Amir Rostami from Universiti Teknologi Petronas, Malaysia, for helping them conducting the CST simulations.

\section{References}

[1] A. Tariq, F. Azam, M. W. Anwar, T. Zahoor, and A. W. Muzaffar, "Recent trends in underwater wireless sensor networks (UWSNs) - a systematic literature review," Programming and Computer Software, vol. 46, no. 8, pp. 699-711, 2020.

[2] J. Heidemann, W. Ye, J. Wills, A. Syed, and Y. Li, "Research challenges and applications for underwater sensor networking," in Proceedings of the IEEE Wireless Communications and Networking Conference, pp. 228-235, WCNC, Las Vegas, NV, USA, April 2006.

[3] M. Rauf, A. M. Khan, A. Ansari, M. T. Jilani, and T. Shahzeb, "Skin depth verification of the electromagnetic waves for hydrocarbon detection," International Journal of Applied Electromagnetics and Mechanics, vol. 60, no. 3, pp. 313-326, 2019.

[4] S. I. Inácio, M. R. Pereira, H. M. Santos et al., "Antenna design for underwater radio communications," in Proceedings of the OCEANS 2016-Shanghai, pp. 1-6, Shanghai, China, April 2016.

[5] R. Islam, R. B. Faruque, and M. Dautta, "Computational investigation of electromagnetic fields in the atmosphere due to electric and magnetic dipoles placed in seawater," in Proceedings of the 2015 International Conference on Electrical \& Electronic Engineering (ICEEE), pp. 201-204, Rajshahi, Bangladesh, November 2015.

[6] M. Dautta, "Computation of electromagnetic field propagation characteristics of a dipole antenna submerged in seawater," in Proceedings of the 2016 International Conference on Wireless Communications, Signal Processing and Networking (WiSPNET), pp. 28-32, Chennai, India, March 2016.

[7] M. Dautta and M. I. Hasan, "Underwater vehicle communication using electromagnetic fields in shallow seas," in Proceedings of the 2017 International Conference on Electrical, Computer and Communication Engineering (ECCE), pp. 3843, Cox's Bazar, Bangladesh, February 2017.

[8] I. I. Smolyaninov, Q. Balzano, C. C. Davis, and D. Young, "Surface wave based underwater radio communication," IEEE Antennas and Wireless Propagation Letters, vol. 17, no. 12, pp. 2503-2507, 2018.

[9] H. Yoshida, "Underwater electromagnetics and its application to unmanned underwater platforms," in Proceedings of the 2016 IEEE International Underwater Technology Symposium (UT), pp. 1-5, Tokyo, Japan, March 2013.

[10] A. Zoksimovski, D. Sexton, M. Stojanovic, and C. Rappaport, "Underwater electromagnetic communications using conduction - channel characterization," Ad Hoc Networks, vol. 34, pp. 42-51, 2015.

[11] S. M. Santhanam, "Study and analysis of various modulation techniques for underwater communication," in Proceedings of the International Conference on Emerging Trends in Science and Engineering, Karnataka, India, May 2017.

[12] J. Lloret, S. Sendra, M. Ardid, and J. J. P. C. Rodrigues, "Underwater wireless sensor communications in the $2.4 \mathrm{GHz}$ 
ISM frequency band," Sensors, vol. 12, no. 4, pp. 4237-4264, 2012.

[13] J. Joe and S. Toh, "Digital underwater communication using electric current method," in Proceedings of the OCEANS 2007Europe, pp. 1-4, Aberdeen, UK, June 2007.

[14] B. Kelley, K. Manoj, and M. Jamshidi, "Broadband RF communications in underwater environments using multicarrier modulation," in Proceedings of the 2009 IEEE International Conference on Systems, Man and Cybernetics, pp. 2303-2308, San Antonio, TX, USA, October 2009.

[15] T. Esemann, G. Ardelt, and H. Hellbrück, "Underwater electric field communication," in Proceedings of the International Conference on Underwater Networks \& Systems, pp. 1-5, Rome, Italy, November 2014.

[16] X. Wu, W. Xue, and X. Shu, "Design and implementation of underwater wireless electromagnetic communication system," in Proceedings of the AIP Conference Proceedings, p. 020022, Chongqing, China, August 2017.

[17] G. Singh and M. Kumar, "Performance analysis of electromagnetic (EM) wave in sea water medium," Wireless Networks, vol. 26, no. 3, pp. 2125-2135, 2020.

[18] C. Yip, A. Goudevenos, and J. Lucas, "Antenna design for the propagation of EM waves in seawater," Underwater Technology, vol. 28, no. 1, pp. 11-20, 2008.

[19] H. Shaikh, M. A. Nadeem, M. Y. Zaheen, A. M. Khan, and M. Rauf, "Underwater channel characterization for effective communication link," in Proceedings of the 3rd International Conference on Computing, Mathematics and Engineering Technologies (iCoMET), pp. 1-5, Sukkur, Pakistan, January 2020.

[20] I. I. Smolyaninov, "Surface electromagnetic waves at gradual interfaces between lossy media," Progress In Electromagnetics Research, vol. 170, pp. 177-186, 2021.

[21] A. Rostami, N. Yahaya, H. Soleimani et al., "Source modification for efficiency enhancement of marine controlledsource electromagnetic method," Journal of Geophysics and Engineering, vol. 18, Article ID gxab011, 2021.

[22] G. Hattab, M. El-Tarhuni, M. Al-Ali, T. Joudeh, and N. Qaddoumi, “An underwater wireless sensor network with realistic radio frequency path loss model," International Journal of Distributed Sensor Networks, vol. 9, no. 3, Article ID 508708, 2013.

[23] Z. Q. Nie, S. L. Wang, D. H. Chen, and T. Deng, "Seabed-rocklayer electromagnetic communication channel model with low path loss based on evanescent wave," Radioengineering, vol. 27, no. 2, pp. 431-439, 2018.

[24] T. Shahzeb, M. Rauf, A. M. Khan, S. M. H. Hashmi, and K. H. Ansari, "Seabed logging validation in a lab scale setup for hydrocarbon exploration," in Proceedings of the 2018 12th International Conference on Mathematics, Actuarial Science, Computer Science and Statistics (MACS), pp. 1-4, Karachi, Pakistan, November 2018.

[25] J. Li, M. Toulgoat, M. Déziel, F. R. Yu, and S. Perras, "Propagation modeling and mac-layer performance in EMbased underwater sensor networks," in Proceedings of the Fourth ACM International Symposium on Development and Analysis of Intelligent Vehicular Networks and Applications, pp. 111-117, Montreal, Canada, September 2014.

[26] E. Jimenez, G. Quintana, P. Mena, P. Dorta, I. Perez-Alvarez, S. Zazo et al.,et al. "Investigation on radio wave propagation in shallow seawater: simulations and measurements," in Proceedings of the 2016 IEEE Third Underwater Communications and Networking Conference (UComms), pp. 1-5, Lerici, Italy, August 2016.
[27] J. I. Nordskag and L. Amundsen, "Asymptotic airwave modeling for marine controlled-source electromagnetic surveying," Geophysics, vol. 72, no. 6, pp. F249-F255, 2007.

[28] Y. Zhou, W. Wang, H. Zhang, C. Wang, G. Xu, and G. Xie, "Communication distance correlates positively with electrode distance in underwater electrocommunication," in Proceedings of the 2018 IEEE International Conference on Robotics and Biomimetics (ROBIO), pp. 88-93, Kuala Lumpur, Malaysia, December 2018.

[29] M. Rauf, M. Aamir, and A. M. Khan, "A prospect of efficient radio-frequency based underwater wireless data transfer," in Proceedings of the Global Conference on Wireless and Optical Technologies (GCWOT), pp. 1-5, Malaga, Spain, October 2020. 